\title{
STP-C, an Oncoprotein of Herpesvirus saimiri Augments the Activation of NF- $k B$ through Ubiquitination of TRAF6
}

\author{
Young-Hwa Chung,*, Byung Hak Jhun ${ }^{1}$, Su-Chak Ryu ${ }^{2}$ Heui-Soo Kim ${ }^{3}$, Cheol-Min Kim ${ }^{4}$, \\ Bong-Seok Kim ${ }^{5}$, Young-Ok Kim ${ }^{5}$ and Sang Jun Lee \\ ${ }^{1}$ Department of Nanomedical Engineering, 2 Department of Nanomaterials Engineering BK21 Nano Fusion Technology Team, \\ Joint Research Center of PNU-Fraunhofer IGB, Pusan National University, Miryang 627-706, Korea \\ ${ }^{3}$ Department of Biology, ${ }^{4}$ Department of Biochemistry, Pusan National University, Busan 609-735, Korea \\ ${ }^{5}$ Biotechnology Research Center, National Fisheries Research \& Development Institute, Busan 619-902, Korea
}

Received 28 September 2006, Accepted 15 December 2006

\begin{abstract}
Herpesvirus saimiri (HVS), a member of the $\gamma$-herpesvirus family, encodes an oncoprotein called Saimiri Transforming Protein (STP) which is required for lymphoma induction in non-human primates. Previous study has shown that STP-C, an oncoprotein of HVS, activates NF- $\kappa$ B signaling pathway. However, the detailed mechanism of STP-Cmediated NF- $\kappa$ B activation has not been reported yet. We first report that STP-C interacts with TRAF6 protein in vivo and in vitro and further investigation shows that $\mathrm{Glu}_{12}$ residue of STP-C is critical for binding to TRAF6. Introduction of ubiquitin together with STP-C augments NF- $\kappa$ B activity compared to that of STP-C expression alone. STP-C expression further induces ubiquitination of endogenous TRAF6. In addition, either a deubiquitination enzyme, CYLD or a dominant negative E2-conjugation enzyme reduced $N F-\kappa B$ activity in spite of the presence of STP-C, supporting that the interaction between STP-C and TRAF6 induces ubiquitination of TRAF6. NF- $\kappa$ B activation by STP-C through the ubiquitinated TRAF6 causes the increased production of IL-8, an inflammatory chemokine and the enhanced expression of costimulatory molecule ICAM, which might ultimately contribute cellular transformation by the exposure of HVS-infected cells with inflammatory microenvironment and chronic activation.
\end{abstract}

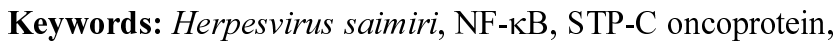
TRAF6, Ubiquitin

*To whom correspondence should be addressed. Tel: 82-55-350-5296; Fax: 82-55-350-5653

E-mail: younghc@pusan.ac.kr

\section{Introduction}

Despite of a structural homology between TNF- $\alpha$ receptor associated factor (TRAF) molecules, each TRAF harbors a distinct biological function involved in cell death, survival, and immune responses (Bradley and Pober, 2001; Chung et al., 2002; Lee and Lee, 2002). TRAF family members 2, 5, and 6 share functions to activate Jun-N terminal kinase (JNK) and p38 MAP kinase (Baud et al., 1999; Dempsey et al., 2003), whereas neither TRAF3 nor TRAF4 activates these pathways (Chung et al., 2002; Ely and Li, 2002). Recent studies have shown that the structural determinant of the TRAF6-binding motif; a PxExxE/Ф (Acidic residue/hydrophobic residue) is distinct from TRAF2-binding motif; a PxQxT/S with crystallization (Ye et al., 2002). Furthermore, gene knockout studies have proposed that TRAF2 is responsible for JNK activity and TRAF6 is critical for NF- $\mathrm{BB}$ signaling (Lomaga et al., 1999; Nguyen et al., 1999). Thus, TRAF6 functions as an adaptor protein for various receptors such as IL-1/Toll-like-, TNF-related activation-induced cytokine (TRANCE)-, and CD40- receptor, leading to NF- $\kappa \mathrm{B}$ activation (Jabara et al., 2002).

Ubiquitin (Ub) is a globular protein with 76 amino acid residues that is highly conserved throughout eukaryotes, with three amino acid changes from yeast to human (Pickart, 2001). The classical view of ubiquitination is that ubiquitination targets proteins for degradation by a multi-subunit, ATPdependent 26 proteosome (Pickart, 2001; Lee and Yu, 2005). Conjugation involves the sequential actions of activating (E1), conjugating (E2), and ligase (E3) enzymes (Pickart, 2001; Pickart, 2004). Poly-ubiquitination chain linked through K48 on $\mathrm{Ub}$ targets a protein for proteosome degradation although poly-chains of Ub can be formed through K11 and K29 in vivo (Chau et al., 1989; Peng et al., 2003). Apparently, poly$\mathrm{Ub}$ chain linked through K63 is not a proteosome-targeting 
signal. Instead, K63 linkage on Ub is important for DNA repair and IKB kinase activation although the detailed mechanism has not been illustrated (Deng et al., 2000; Hoege et al., 2002).

Due to the induction of lymphoma in New World primates (Jung et al., 1999; Damania et al., 2000), studies on Herpesvirus saimiri (HVS), a member of $\gamma$-herpesvirus family have been extensively performed as an alternative animal model for studies on Epstein Barr Virus (EBV)- or Kaposi's sarcomaassociated herpesvirus (KSHV)-mediated pathogenic disease of human (Jung and Desrosiers, 1991; Jung and Desrosiers, 1995). HVS can be further subclassified into three subgroups (HVS-A, B, and C) on the basis of the extent of DNA sequence divergence at the left end of L-DNA (Medveczky et al., 1984). Thus, mutational analyses on the gene products designated with saimiri transforming protein (STP-A, or C) have demonstrated that STP is not required for viral replication but for immortalization (Murthy et al., 1989). Further studies on STP-C-mediated oncogenesis have revealed that STP-C interacts with not only oncogene Ras molecule, leading to MAP kinase activation (Jung and Desrosiers, 1995) and but also TRAF2, 3 and 5 molecules for NF- $\mathrm{KB}$ activation (Lee et al., 1999).

Although previous study has shown that STP-C activates NF- $\kappa \mathrm{B}$ signaling pathway (Lee et al., 1999), the detailed mechanism of STP-C-mediated NF- $\mathrm{BB}$ activation has not been reported yet. Herein, we first report that STP-C binds to TRAF6 in vivo and in vitro, leading to NF- $\kappa \mathrm{B}$ activation. The expression of STP-C induced ubiquitination of endogenous TRAF6. Blockage of TRAF6 ubiquitination with CYLD, a deubiquitination enzyme or a dominant negative-E2 conjugation enzyme; Ubc13 (C87A) drastically diminished STP-C-mediated $\mathrm{NF}-\kappa \mathrm{B}$ activation. Finally, STP-C expression induced the production of IL-8 and ICAM expression through TRAF6 ubiquitination, which might ultimately contribute cellular transformation by the exposure of HVS-infected cells with inflammatory microenvironment and chronic activation.

\section{Materials and Methods}

Cell culture and transfection. Human embryonic kidney (HEK) 293 T cells, Flp-In Trex-293 cells expressing the tetracycline repressor protein (Invitrogen), and Hela cells were cultured in DMEM supplemented with $10 \%$ FBS and $1 \%$ penicillin and streptomycin. BJAB B lymphocytes and Jurkat $\mathrm{T}$ lymphocytes were cultured in RPMI-1640 supplemented with $10 \%$ FBS and $1 \%$ penicillin and streptomycin. HEK 293 T cells and Hela cells were plated at $5 \times 10^{6}$ per 100 -mm-diameter plate $24 \mathrm{~h}$ before transfection and the cells were transfected with 8 to $15 \mathrm{mg}$ of DNA using calcium phosphate precipitation method (Clontech) and Lipofectamin 2000 (Invitrogen), respectively. BJAB B and Jurkat T cells were electroporated with $10 \mu \mathrm{g}$ of DNA at $250 \mu \mathrm{F}, 950 \mathrm{~V}$ and cultured for $48 \mathrm{~h}$ for the measurement of surface expression of ICAM.

Plasmid construction. The STP-C gene was amplified by PCR and subcloned into the pEF1 vector (Invitrogen). $\mathrm{Glu}_{12}$ residue of STP-C was substituted with Ala using PCR and designated as E12A. E12A mutant was subcloned to the pEF1 vectors. To make Flp-In Trex-293 cells expressing tetracycline-inducible wild type (WT) or E12A mutant of STP-C, WT and E12A genes of STP-C from the pEF-1 were excised and inserted into pCDNA5/FRT/To vector (Invitrogen). To examine the effect of ICAM expression by the expression of WT and E12A mutant of STP-C, WT and E12A genes from the $\mathrm{pEF}-1$ were excised and inserted to $\mathrm{pTracerA}$ vector (Invitrogen). To construct glutathione S-transferase (GST) fusion recombinant DNA, STP-C fragment was amplified by PCR and inserted into pGEX4T-1 vector (Amersham).

Immunoprecipitation and immunoblotting. Cells were harvested and lysed with lysis buffer $(0.15 \mathrm{M} \mathrm{NaCl}, 1 \%$ Nonidet P-40, 50 $\mathrm{mM}$ Tris[pH7.5]) containing $0.1 \mathrm{mM} \mathrm{Na} \mathrm{VO}_{3}, 1 \mathrm{mM} \mathrm{NaF}$, and protease inhibitors (leupeptin, aprotinin, phenylmethylsulfonyl fluoride, and bestatin). For immunoblotting, proteins from whole cell lysate were resolved by $10 \%$ or $12 \%$ SDS-PAGE and transferred to nitrocellulose membranes. Primary antibodies were used at $1: 1,000$ or $1: 2,000$ dilutions, and secondary antibodies were used at $1: 2,000$ dilution $6 \%$ nonfat dry milk. After final washing, nitrocellulose membranes were exposed for an enhanced chemiluminescence assay.

In vitro GST pull-down assay. The GST and GST-STP-C recombinant protein were purified from Escherichia coli BL21 (Invitrogen) with glutathione Sepharose 4B beads as recommended by the manufacturer (Amersham). At $48 \mathrm{~h}$ after transfection with TRAF6 vector, HEK 293 T cells were lysed with binding buffer (20 $\mathrm{mM}$ Tris [pH 7.5], $150 \mathrm{mM} \mathrm{NaCl}, 1 \%$ Triton X-100, 1\% Protease inhibitor cocktail solution (Sigma-Aldrich)) and mixed with $10 \mu \mathrm{g}$ of the GST fusion protein for $2 \mathrm{~h}$ at $4^{\circ} \mathrm{C}$. Then, glutathione Sepharose 4B beads were extensively washed and subjected to $10 \%$ SDSPAGE, followed by an immunoblot assay.

NF- $\kappa$ B reporter assay. HEK $293 \mathrm{~T}$ cells or Hela cells were transfected with WT or its mutant STP-C vector together with NF$\kappa \mathrm{B}$-luciferase report vector by the calcium phosphate precipitation method or Lipofectamin 2000 (Invitrogen). To normalize transfection efficiency, the pGK- $\beta$ gal vector that expresses $\beta$-galactosidase from a phosphoglucokinase promoter, was included in the transfection mixture. At $48 \mathrm{~h}$ post-transfection, cells were washed with cold phosphate-buffered saline (PBS) and lysed in lysis solution ( $25 \mathrm{mM}$ Tris[pH7.8], $2 \mathrm{mM}$ EDTA, $2 \mathrm{mM}$ DTT, 10\% glycerol, and 1\% Triton X-100). Luciferase activity was measured with a luminometer by using a luciferase assay kit (Promega).

Treatment of siRNA against TRAF6. Hela cells were plated at equal densities in 6 well plate $\left(2 \times 10^{5}\right.$ cells/well $)$ and treated with siRNAs against TRAF6 or a nonspecific control at $24 \mathrm{~h}$ after transfection with STP-C and NF- $\mathrm{KB}$ reporter vectors using XtremeGene siRNA transfection reagent (Roche) as described by the manufacturer's instruction. Sequences used were as follows. For the control, siRNA control; 5'-CCU ACG CCA CCA AUU UCG U-3' as sense and 5'-ACG AAA UUG GUG GCG UAG G-3' as antisense were synthesized and for TRAF6, the siRNAs were used; 5'-CUG UGC UGC AUC AAU GGC A-3' as sense and 5'-UGC 
CAU UGA UGC AGC ACA G-3' as antisense. The cells were harvested for immunoblotting and NF- $\mathrm{KB}$ reporter assay at $24 \mathrm{~h}$ after siRNA treatment.

Flow cytometry. BJAB B cells or Jurkat T cells $\left(1 \times 10^{6}\right.$ cells $)$ were washed with RPMI-1640 medium supplemented with $10 \%$ FBS and stained with a phycoerythrin(PE)-conjugated ICAM monoclonal Antibody (Becton Dickson) at $4^{\circ} \mathrm{C}$ for $30 \mathrm{~min}$. After washing with RPMI-1640 medium supplemented with 10\% FBS, and the flow cytometry was performed by FACS (Becton Dickson).

Enzyme-liked immunosorbent assay (ELISA). At $48 \mathrm{~h}$ after induction of WT or mutant STP-C expression by deoxycycline (2 $\mu \mathrm{g} / \mathrm{ml})$, the cell supernatants were harvested and used for the measurement of IL-8 production using Pharmingen ELISA kit (Becton Dickson)

\section{Results}

Enhanced polyubiquitination due to presence of STP-C augments NF- $\kappa \mathbf{B}$ activity. Recently, it has been reported that a poly-ubiquitination of a protein indicates not only protein degradation in the $26 \mathrm{~S}$ proteosome but also activation of NF$\kappa \mathrm{B}$ (Deng et al., 2000; Goldberg, 2003). Expression of STP$\mathrm{C}$, an oncoprotein of HVS has been shown to increase NF- $\mathrm{KB}$ activity (Lee et al., 1999). These series of evidence prompted us to explore whether expression of STP-C induces polyubiquitination of proteins, leading to activation of NF- $\mathrm{BB}$. First of all, to examine whether presence of STP-C induces poly-ubiquitination of proteins HEK 293T cells were transfected with Ubiquitin ( $\mathrm{Ub})$ expression vector along with or without STP-C expression vector. As shown in Fig. 1A, the expression of $\mathrm{Ub}$ alone induced a weak poly-ubiquitination of cellular proteins, which is further enhanced by co-expression of $\mathrm{Ub}$ and STP-C. Co-expression of $\mathrm{Ub}$ and protein-linking integrinassociated protein and cytoskeleton (PLIC-1), a cargo protein involved in a link between the ubiquitinated protein(Kneussel, 2002) and the proteosome machinery, also increased polyubiquitination as seen in co-expression of $\mathrm{Ub}$ and STP-C.

Next, when NF- $\kappa \mathrm{B}$ activity was examined in the expression of STP-C or PLIC-1 together with Ub, co-expression of STP$\mathrm{C}$ and $\mathrm{Ub}$ increased a drastic activity of NF- $\mathrm{BB}$, whereas coexpression of PLIC-1 and $\mathrm{Ub}$ did not affect any activity of NF- $\kappa \mathrm{B}$ (Fig. 1B). This result implies that poly-ubiquitination by the presence of STP-C is qualitatively different from that by the presence of PLIC-1. Of interest, co-expression of STP$\mathrm{C}$ and $\mathrm{Ub}$ showed much higher activity of $\mathrm{NF}-\kappa \mathrm{B}$, compared to that of STP-C expression alone. The result suggests that an enhanced poly-ubiquitination due to expression of STP-C augments NF- $\kappa \mathrm{B}$ activity.

STP-C interacts with TRAF6 in vivo and in vitro. Because STP-C has been reported to interact with TRAF2, 3, and 5 using the binding motif of PxQxT/S (Lee et al., 1999), we examined whether STP-C carries TRAF6 binding motif such as PxExxE/Ф or not. We found that STP-C oncoprotein indeed contains a candidate TRAF6-binding motif; $\mathrm{P}_{11}$ IEETGD $_{17}$ although it is not exactly matched to the published one (Ye et al., 2002). Therefore, we pursued whether STP-C oncoptotein interacts with TRAF6 in vivo and in vitro. HEK 293 T cells were transfected with STP-C, TRAF6 or both STP-C and TRAF6, and the cell lysates were immunoprecipitated with anti-STP-C (109) antibodies (Jung and Desrosiers, 1995) and blotted with anti-flag antibody for the detection of TRAF6. TRAF6 protein was only detected in the co-expression of STP-C and TRAF6 (Fig. 2A), indicating that STP-C interacts

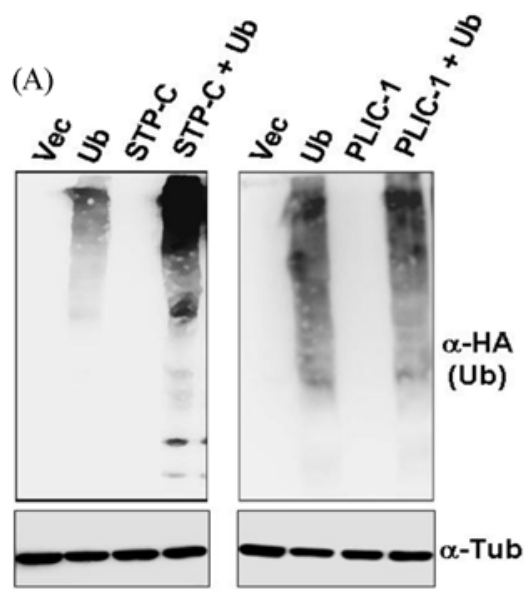

(B)

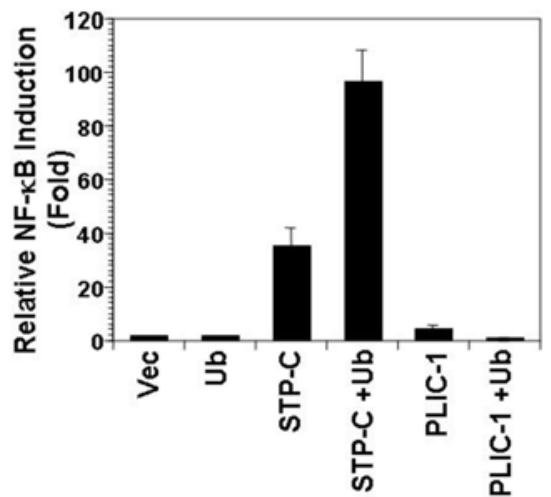

Fig. 1. Augmentation of NF- $\mathrm{KB}$ activity by enhanced ubiquitination due to the expression of STP-C. (A) After pEF1-STP-C and pCMV4-Flag-PLIC-1 with or without pEF-HA-Ub were transfected into HEK 293T cells, the cell lysates of ubiquitination were detected with an anti-HA antibody. $\beta$-Tubulin was used for the loading control. (B) After transfection with STP-C (3 $\mu$ g) and PLIC-1 $(3 \mu \mathrm{g})$ in the presence or absence of Ub $(2 \mu \mathrm{g})$, NF- $\kappa \mathrm{B}$ luciferase activity was measured. Transfection efficiency was normalized with $\beta$-galactosidase activity. 


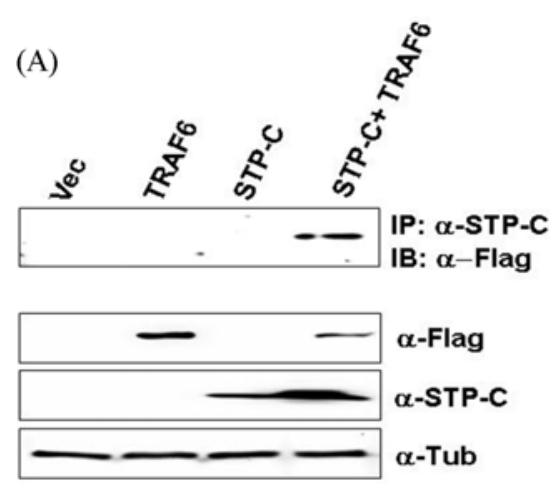

(C)

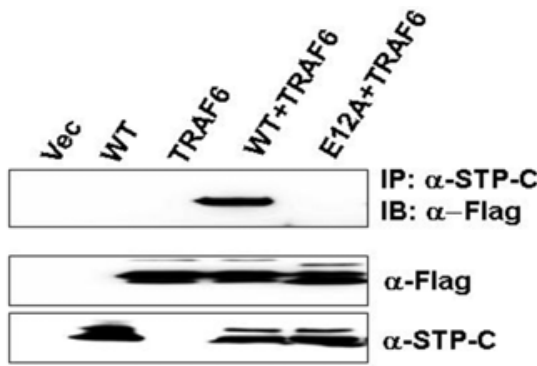

(B)
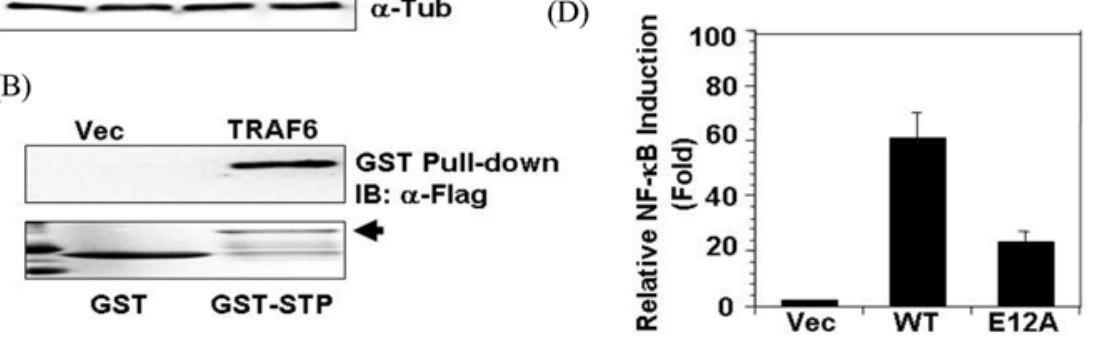

Fig. 2. Interaction of STP-C with TRAF6 in vivo and vitro. (A) After STP-C, TRAF6 (pCDNA3-Flag-TRAF6) or both were expressed, cell lysates were used for immunoprecipitation (IP) with rabbit anti-STP-C antibodies, followed by IB with an anti-flag antibody for detection of TRAF6. (B) GST or GST -STP-C protein was mixed with cell lysates containing flag-tagged TRAF6. GST bead complexes were separated on 10\% SDS-PAGE and TRAF6 protein was detected with an anti-flag antibody. (C) After wild type (WT) of STP-C and E12A mutant with or without TRAF6 were expressed, cell lysates were used for IP with an anti-flag antibody for precipitation of TRAF6, followed by IB with rabbit anti-STP-C antibodies. (D) After transfection with WT (3 $\mu$ g) or its mutant E12A $(3 \mu \mathrm{g})$ of STP-C, NF- $\mathrm{KB}$ luciferase activity was measured. Transfection efficiency was normalized with $\beta$-galactosidase activity

with TRAF6 in vivo. In addition, to examine whether STP-C interacts with TRAF6 in vitro, GST and GST-STP-C recombinant protein were expressed and purified as described previously (Lee et al., 1999). TRAF6 from the cell lysate was pulled down with GST-STP-C or GST. As seen in Fig. 2B, GST-STP-C did precipitate TRAF6 whereas GST did not. Therefore, the results indicate that STP-C specifically binds to TRAF6 in vivo and in vitro.

Furthermore, in order to pursue the search of a crucial residue in STP-C for the interaction with TRAF6, Glu 12 residue of STP-C was substituted with Ala, designated as E12A.We performed an immunoprecipitation with anti-STPC antibodies using cell lysates of co-transfection with TRAF6 and WT, or E12A mutant of STP-C, and thereafter followed an immunoblotting with anti-flag antibody for the detection of TRAF6. WT of STP-C interacted with TRAF6 but E12A mutant lost the ability of binding to TRAF6 (Fig. 2C). The result clearly exhibited that $\mathrm{Glu}_{12}$ residue of STP-C is necessary for binding to TRAF6. The importance of the interaction between STP-C and TRAF6 was thereafter addressed. WT or E12A of STP-C was transfected with NF$\kappa \mathrm{B}$ reporter vector and measured for $\mathrm{NF}-\kappa \mathrm{B}$ activity. E12A mutant of STP-C, losing the binding ability of TRAF6 exhibited a drastically reduced NF- $\kappa \mathrm{B}$ activity, compared to that of WT (Fig. 2D). Therefore, the results indicate that $\mathrm{Glu}_{12}$ residue of STP-C is important for the interaction with TRAF6 and $\mathrm{NF}-\kappa \mathrm{B}$ activation.
Ubiquitination of TRAF6 by the expression of STP-C is critical for the activation of $N F-\kappa B$. Because TRAF6 protein contains Zinc-finger and RING finger domains at the $\mathrm{N}$-terminus and TRAF C domain at the C-terminus as similar as structures of the other TRAF proteins (Bradley and Pober, 2001; Wajant and Scheurich, 2001; Chung et al., 2002) and has been known to act as an E3 Ub ligase (Wang et al., 2001), we explored a role of RING finger motif for the ubiquitination. An extensive ubiquitination of WT TRAF6 was detected in the co-expression of $\mathrm{Ub}$ and TRAF6, whereas ubiquitination of TRAF6 mutant, depleted of RING finger domain did not occur. The result indicates that RING finger domain is involved in the ubiquitination of TRAF6, which is critical for E3 Ub ligase activity (Fig. 3A). Furthermore to examine whether the presence of STP-C induces ubiquitination of endogenous TRAF6, HEK 293T cells were co-transfected with STP-C and Ub vectors. Endogenous TRAF6 was immunoprecipited using anti-TRAF6 antibody from the cell lysate and ubiquitination of endogenous TRAF6 was detected with anti-HA antibody because of HA-tagged Ub vector. As shown in Fig. 3B, ubiquitination of endogenous TRAF6 in the absence of STP-C was hardly detected from TRAF6 immune complex but ubiquitination of endogenous TRAF6 was detected from TRAF6 immune complex in the presence of STP-C. The result proposes that STP-C increases ubiquitination of TRAF6, resulting in enhancement of NF- $\kappa$ B activity. Furthermore, we attempted to suppress endogenous TRAF6 

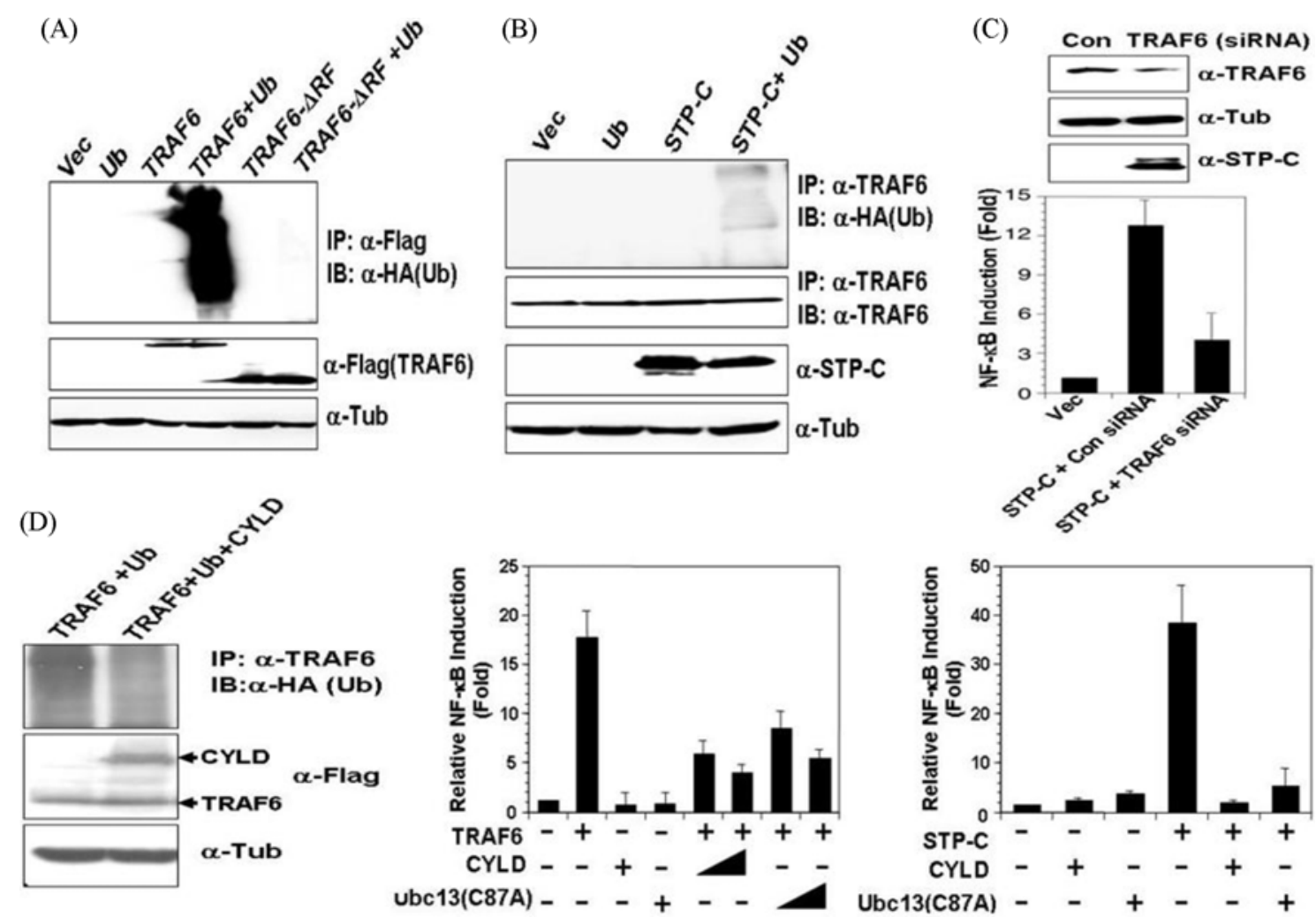

Fig. 3. A critical role of ubiquitinated TRAF6 through the expression of STP-C in NF- $\kappa B$ activation. (A) After TRAF6 and a dominant negative (DN)-TRAF6, depleted of RING finger motif (pCDNA3-Flag-TRAF6- $\triangle R F$ ) with or without Ub were co-expressed, the cell lysates were used for IP with an anti-flag antibody for precipitation of TRAF6, followed by IB with an anti-HA antibody for the detection of ubiquitination. (B) After STP-C in the presence or absence of Ub was expressed, the cell lysates were used for IP with anti-TRAF6 antibodies, followed by IB with an anti-HA antibody for the detection of ubiquitination. (C) After Hela cells were transfected with STP-C $(2 \mu \mathrm{g})$ and NF- $\mathrm{KB}$ reporter vector $(1 \mu \mathrm{g})$, endogenous TRAF6 expression was suppressed using its siRNA $(1.5 \mu \mathrm{g})$. The suppressed TRAF6 identified by IB with anti-TRAF6 antibodies. NF- $\kappa B$ luciferase activity was measured and transfection efficiency was normalized with $\beta$-galactosidase activity. (D) After flag-tagged TRAF6 $(2 \mu \mathrm{g})$ and HA-tagged Ub $(2 \mu \mathrm{g})$ were expressed with or without CYLD (pCDNA3-Flag-CYLD; $4 \mu \mathrm{g}$ ), the cell lysates used for IP with anti-TRAF6 antibodies, followed by IB with an anti-HA antibody for the detection of ubiquitination. When TRAF6 $(2 \mu \mathrm{g})$ or STP-C $(2 \mu \mathrm{g})$ was expressed together with CYLD $(0.5$ and $2 \mu \mathrm{g}$ ) or Ubc13 (C87A) (pEF-HA-Ubc13 (C87A); 0.5 and $2 \mu \mathrm{g}$ ), NF- $\mathrm{kB}$ luciferase activity was measured. Transfection efficiency was normalized with $\beta$-galactosidase activity.

expression using its siRNA to clarify a role of TRAF6 in STP$\mathrm{C}$-mediated NF- $\mathrm{BB}$ activation. When the expression of TRAF6 was inhibited by its siRNA, we found significantly reduced NF- $\kappa$ B activity in spite of the presence of STP-C (Fig. 3C).

To test our proposal that increased ubiquitination of TRAF6 due to the expression of STP-C augments NF- $\mathrm{B}$ activity, we examined whether deubiquitination of TRAF6 by the expression of CYLD reduces NF- $\kappa \mathrm{B}$ activity in spite of the presence of STP-C (Kovalenko et al., 2003; Trompouki et al., 2003). First, we identified that the expression of CYLD inhibits ubiquitination of TRAF6 (Fig. 3D) as reported previously (Kovalenko et al., 2003; Trompouki et al., 2003). TRAF6 expression alone enhanced NF- $\mathrm{BB}$ activity, whereas coexpression of TRAF6 and CYLD reduced NF- $\kappa \mathrm{B}$ activity (Fig. 3C). Furthermore, expression of Ubc13 (C87A), a dominant negative E2 conjugation enzyme; Ubc13/Uev1A
(Deng et al., 2000; Wang et al., 2001), also decreased TRAF6-mediated NF- $\kappa$ B activity (Fig. 3D). Introduction of either CYLD or Ubc13 (C87A) in STP-C expression drastically diminished NF- $\kappa$ B activity as seen in TRAF6 expression (Fig. 3D). Therefore, the result supports that increased ubiquitination of TRAF6 due to the expression of STP-C augments NF- $\kappa B$ activity.

Interaction of STP-C with TRAF6 is important for the production of IL-8 and the expression of ICAM. To examine a biological role of the interaction of STP-C with TRAF6, we generated 293 cell lines expressing tetracyclineinducible WT or E12A mutant of STP-C and transiently induced the expression of WT or E12A mutant of STP-C in BJAB B and Jurkat T lymphocytes. When doxycycline, an analogue of tetracycline was added, the expression of WT STP-C dramatically increased NF- $\mathrm{B}$ activity while the 
(A)

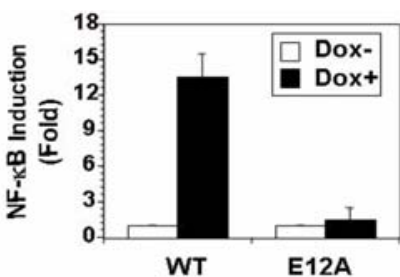

(C)

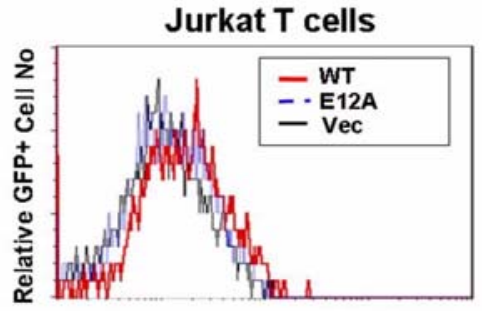

(B)

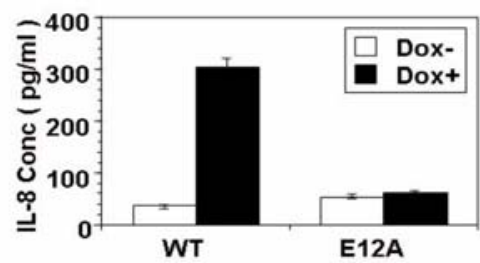

BJAB cells

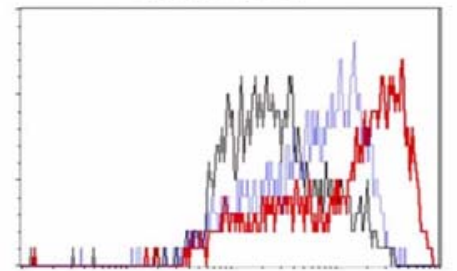

Expression of ICAM

Fig. 4. Production of IL-8 and expression of ICAM through interaction of STP-C with TRAF6. (A) Doxycycline $(2 \mu \mathrm{g} / \mathrm{ml})$ was added to Flp-In Trex 293 cells for $24 \mathrm{~h}$ to induce WT or E12A of STP-C, and NF- $\kappa B$ luciferase activity was measured. Transfection efficiency was normalized with b-galactosidase reporter vector pGK- $\beta$ gal. (B) Doxycycline was added to Flp-In Trex 293 cells for $48 \mathrm{~h}$ to induce WT or E12A of STP-C and the supernatant was harvested, followed by measurement of IL-8 with an ELISA kit. (C) Jurkat $\mathrm{T}$ or BJAB cells $\left(1 \times 10^{7}\right.$ cells $)$ were electroporated with pTracer vector expressing WT, or E12A mutant of STP-C and incubated for $48 \mathrm{~h}$. The cells were washed and stained PE-conjugated anti- ICAM antibody, followed by the measurement of ICAM expression on the GFP positive gate with a flow cytometry.

expression of E12A mutant did not (Fig. 4A). This result was exactly correlated with IL-8 production; the expression of STP-C produced a significant amount of IL-8, whereas the expression of E12A mutant did not. The result indicates that interaction of STP-C with TRAF6 is important for the production of IL-8, an inflammatory chemokine and angiogenesis factor.

WT or E12A mutant of STP-C, subcloned to pTracer vector carrying GFP was transfected to BJAB B and Jurkat T lymphocytes, and ICAM expression was measured among GFP positive cell populations using FACS. As seen in Fig. 4B, the transient expression of WT STP-C induced ICAM expression on $\mathrm{BJAB}$ and Jurkat $\mathrm{T}$ cells. However, the expression of E12A mutant reduced the level of ICAM expression, compared to that of WT STP-C. These results suggest that NF- $\kappa \mathrm{B}$ activation by the expression of STP-C through TRAF6 plays a crucial role in the production of an angiogenesis factor and a costimulatory molecule.

\section{Discussion}

In addition to the role of $\mathrm{Ub}$ in protein degradation, $\mathrm{Ub}$ plays an essential role in IKK activation by TRAF proteins (Deng et al., 2000; Shi and Kehrl, 2003). During cytokine signaling such as TNF- $\alpha$ and IL-1 $\beta$, TRAF2 and TRAF6 are ubiquitinated and they act as E3 ligases that function together with Ubc13/Uev1A as a conjugation enzyme to catalyze the synthesis of a unique poly-Ub chain linked through K63 of Ub. Furthermore, it has been reported that ubiquitinated TRAF2 and TRAF6 are pre-requisite for NF- $\kappa$ B signaling
(Deng et al., 2000; Shi and Kehrl, 2003; Habelhah et al., 2004). Herein, we observed that STP-C expression induces poly-ubiquitination of endogenous TRAF6 as seen in cytokine IL-1 $\beta$ signaling. We thus believe that poly-ubiquitinated TRAF6 induced by STP-C facilitates to deliver a signal to a down-stream molecule such as TAK1, resulting in NF- $\mathrm{NB}$ activation. Therefore, we propose that STP-C acts like a TNF receptor superfamily, particularly IL-1 $\beta /$ Toll-like receptor with a ligand-independent manner, which leads to a constitutive

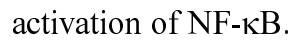

Importance of ubiquitinated TRAF2 or TRAF6 during cytokine signaling has been addressed by CYLD, a tumor suppressor cylindromatosis protein, which acts as a deubiquitination enzyme. The previous studies demonstrate that CYLD facilitates the disassembly of K63-linked poly-Ub chains on TRAF2 and TRAF6, resulting in the inhibition of IKK and subsequent NF- $\mathrm{BB}$ activation by agents such as TNF- $\alpha$, IL-1 and phorbol ester (Kovalenko et al., 2003; Trompouki et al., 2003). We indeed observed that introduction of CYLD inhibits STP-C-mediated NF- $\kappa \mathrm{B}$ activation. Accordingly, we propose that ubiquitination of TRAF6 is necessary for the NF- $\kappa$ B activation mediated by STP-C. However, due to a result of a long exposure of the blot to detect ubiquitination of TRAF6 in Fig. 3B, we could not exclude a possibility that a small portion of ubiquitinated TRAF6 might lead to its degradation and recycling, which may be undetectable with a conventional method. Therefore, a detailed biochemical mechanism for poly-ubiquitination of TRAF6 by STP-C remains as assignment to be resolved.

It is interesting that $\mathrm{NF}-\kappa \mathrm{B}$ is a common target for 
oncogenic viral proteins including Tax protein of human $\mathrm{T}$ lymphocytic leukemia virus (HTLV)-1, Core protein of Hepatitis C virus, and latent membrane protein (LMP)-1 of EBV (Kaye et al., 1996; Yin et al., 1998; Yoshida et al., 2001). In addition, an elevated NF- $\kappa B$ expression and inflammatory cytokines have been frequently found in clinical tumor tissues (Wissink et al., 1997; Griffin, 2001; Helbig et $a l ., 2003)$. Cancerous cells furthermore migrate into vessel walls through various adhesion molecules including ICAM-1, VCAM-1, and ELAM-1, which are up-regulated by NF-кB expression (van de Stolpe and van der Saag, 1996). We herein showed that activated NF- $\kappa$ B by STP-C induces to increase the expression of IL-8 and ICAM. We thus propose that elevated NF- $\mathrm{BB}$ induces the extension of host cell survivals, upon HVS infection into host cells. HVS-infected cells are further exposed under a chronic inflammatory environment and continuous activation through costimulatory molecules with ligand independent manner, which may ultimately contribute cellar transformation.

Acknowledgments We thank Dr. Jae Jung (Harvard Medical School, USA) for valuable comments and materials. This work was supported by 2006 PNU-IGB Joint Research Center Grant of Pusan National University.

\section{References}

Baud, V., Liu, Z. G., Bennett, B., Suzuki, N., Xia, Y. and Karin, M. (1999) Signaling by proinflammatory cytokines: oligomerization of TRAF2 and TRAF6 is sufficient for JNK and IKK activation and target gene induction via an aminoterminal effector domain. Genes Dev. 13, 1297-1308.

Bradley, J. R. and Pober, J. S. (2001) Tumor necrosis factor receptor-associated factors (TRAFs). Oncogene 20, 6482-6491.

Chau, V., Tobias, J. W., Bachmair, A., Marriott, D., Ecker, D. J., Gonda, D. K. and Varshavsky, A. (1989) A multiubiquitin chain is confined to specific lysine in a targeted short-lived protein. Science 243, 1576-1583.

Chung, J. Y., Park, Y. C., Ye, H. and Wu, H. (2002) All TRAFs are not created equal: common and distinct molecular mechanisms of TRAF-mediated signal transduction. J. Cell Sci. 115, 679-688.

Damania, B., Choi, J. K. and Jung, J. U. (2000) Signaling activities of gammaherpesvirus membrane proteins. J. Virol. 74, 1593-1601.

Dempsey, P. W., Doyle, S. E., He, J. Q. and Cheng, G. (2003) The signaling adaptors and pathways activated by TNF superfamily. Cytokine Growth Factor Rev. 14, 193-209.

Deng, L., Wang, C., Spencer, E., Yang, L., Braun, A., You, J., Slaughter, C., Pickart, C. and Chen, Z. J. (2000) Activation of the IkappaB kinase complex by TRAF6 requires a dimeric ubiquitin-conjugating enzyme complex and a unique polyubiquitin chain. Cell 103, 351-361.

Ely, K. R. and Li, C. (2002) Structurally adaptive hot spots at a protein interaction interface on TRAF3. J. Mol. Recognit. 15, 286-290.

Goldberg, A. L. (2003) Protein degradation and protection against misfolded or damaged proteins. Nature 426, 895-899.

Griffin, J. D. (2001) Leukemia stem cells and constitutive activation of NF-kappaB. Blood 98, 2291.

Habelhah, H., Takahashi, S., Cho, S. G., Kadoya, T., Watanabe, T. and Ronai, Z. (2004) Ubiquitination and translocation of TRAF2 is required for activation of JNK but not of p38 or NF-kappaB. EMBO J. 23, 322-332.

Helbig, G., Christopherson, K. W., 2nd, Bhat-Nakshatri, P., Kumar, S., Kishimoto, H., Miller, K. D., Broxmeyer, H. E. and Nakshatri, H. (2003) NF-kappaB promotes breast cancer cell migration and metastasis by inducing the expression of the chemokine receptor CXCR4. J. Biol. Chem. 278, 21631-21638.

Hoege, C., Pfander, B., Moldovan, G. L., Pyrowolakis, G. and Jentsch, S. (2002) RAD6-dependent DNA repair is linked to modification of PCNA by ubiquitin and SUMO. Nature 419, 135-141.

Jabara, H., Laouini, D., Tsitsikov, E., Mizoguchi, E., Bhan, A., Castigli, E., Dedeoglu, F., Pivniouk, V., Brodeur, S. and Geha, R. (2002) The binding site for TRAF2 and TRAF3 but not for TRAF6 is essential for CD40-mediated immunoglobulin class switching. Immunity 17, 265-276.

Jung, J. U., Choi, J. K., Ensser, A. and Biesinger, B. (1999) Herpesvirus saimiri as a model for gammaherpesvirus oncogenesis. Semin. Cancer Biol. 9, 231-239.

Jung, J. U. and Desrosiers, R. C. (1991) Identification and characterization of the herpesvirus saimiri oncoprotein STPC488. J. Virol. 65, 6953-6960.

Jung, J. U. and Desrosiers, R. C. (1995) Association of the viral oncoprotein STP-C488 with cellular ras. Mol. Cell. Biol. 15, 6506-6512.

Kaye, K. M., Devergne, O., Harada, J. N., Izumi, K. M., Yalamanchili, R., Kieff, E. and Mosialos, G. (1996) Tumor necrosis factor receptor associated factor 2 is a mediator of NF-kappa B activation by latent infection membrane protein 1, the Epstein-Barr virus transforming protein. Proc. Natl. Acad. Sci. USA 93, 11085-11090.

Kneussel, M. (2002) Dynamic regulation of GABA(A) receptors at synaptic sites. Brain Res. Rev. 39, 74-83.

Kovalenko, A., Chable-Bessia, C., Cantarella, G., Israel, A., Wallach, D. and Courtois, G. (2003) The tumour suppressor CYLD negatively regulates NF-kappaB signalling by deubiquitination. Nature 424, 801-805.

Lee, C. and Yu, M. H. (2005) Protein folding and diseases. $J$. Biochem. Mol. Biol. 38, 275-280

Lee, H., Choi, J. K., Li, M., Kaye, K., Kieff, E. and Jung, J. U. (1999) Role of cellular tumor necrosis factor receptorassociated factors in NF-kappaB activation and lymphocyte transformation by herpesvirus Saimiri STP. J. Virol. 73, 39133919.

Lee, N. K. and Lee, S. Y. (2002) Modulation of life and death by tumor necrosis factor-receptor-associated factors (TRAFs). $J$. Biochem. Mol. Biol. 35, 61-66.

Lomaga, M. A., Yeh, W. C., Sarosi, I., Duncan, G. S., Furlonger, C., Ho, A., Morony, S., Capparelli, C., Van, G., Kaufman, S., van der Heiden, A., Itie, A., Wakeham, A., Khoo, W., Sasaki, T., Cao, Z., Penninger, J. M., Paige, C. J., Lacey, D. L., Dunstan, C. R., Boyle, W. J., Goeddel, D. V. and Mak, T. W. (1999) TRAF6 deficiency results in osteopetrosis and defective interleukin-1, CD40, and LPS signaling. Genes Dev. 13, 10151024. 
Medveczky, P., Szomolanyi, E., Desrosiers, R. C. and Mulder, C. (1984) Classification of herpesvirus saimiri into three groups based on extreme variation in a DNA region required for oncogenicity. J. Virol. 52, 938-944.

Murthy, S. C., Trimble, J. J. and Desrosiers, R. C. (1989) Deletion mutants of herpesvirus saimiri define an open reading frame necessary for transformation. J. Virol. 63, 3307-3314.

Nguyen, L. T., Duncan, G. S., Mirtsos, C., Ng, M., Speiser, D. E., Shahinian, A., Marino, M. W., Mak, T. W., Ohashi, P. S. and Yeh, W. C. (1999) TRAF2 deficiency results in hyperactivity of certain TNFR1 signals and impairment of CD40-mediated responses. Immunity 11, 379-389.

Peng, J., Schwartz, D., Elias, J. E., Thoreen, C. C., Cheng, D., Marsischky, G., Roelofs, J., Finley, D. and Gygi, S. P. (2003) A proteomics approach to understanding protein ubiquitination. Nat. Biotechnol. 21, 921-926.

Pickart, C. M. (2001) Mechanisms underlying ubiquitination. Annu. Rev. Biochem. 70, 503-533.

Pickart, C. M. (2001) Ubiquitin enters the new millennium. Mol. Cell 8, 499-504.

Pickart, C. M. (2004) Back to the future with ubiquitin. Cell 116, 181-190.

Shi, C. S. and Kehrl, J. H. (2003) Tumor necrosis factor (TNF)induced germinal center kinase-related (GCKR) and stressactivated protein kinase (SAPK) activation depends upon the E2/E3 complex Ubc13-Uev1A/TNF receptor-associated factor 2 (TRAF2). J. Biol. Chem. 278, 15429-15434.

Trompouki, E., Hatzivassiliou, E., Tsichritzis, T., Farmer, H., Ashworth, A. and Mosialos, G. (2003) CYLD is a deubiquitinating enzyme that negatively regulates NF-kappaB activation by TNFR family members. Nature 424, 793-796.

van de Stolpe, A. and van der Saag, P. T. (1996) Intercellular adhesion molecule-1. J. Mol. Med. 74, 13-33.

Wajant, H. and Scheurich, P. (2001) Tumor necrosis factor receptor-associated factor (TRAF) 2 and its role in TNF signaling. Int. J. Biochem. Cell Biol. 33, 19-32.

Wang, C., Deng, L., Hong, M., Akkaraju, G. R., Inoue, J. and Chen, Z. J. (2001) TAK1 is a ubiquitin-dependent kinase of MKK and IKK. Nature 412, 346-351.

Wissink, S., van de Stolpe, A., Caldenhoven, E., Koenderman, L. and van der Saag, P. T. (1997) NF-kappa B/Rel family members regulating the ICAM-1 promoter in monocytic THP-1 cells. Immunobiology 198, 50-64.

Ye, H., Arron, J. R., Lamothe, B., Cirilli, M., Kobayashi, T., Shevde, N. K., Segal, D., Dzivenu, O. K., Vologodskaia, M., Yim, M., Du, K., Singh, S., Pike, J. W., Darnay, B. G., Choi, Y. and Wu, H. (2002) Distinct molecular mechanism for initiating TRAF6 signalling. Nature 418, 443-447.

Yin, M. J., Christerson, L. B., Yamamoto, Y., Kwak, Y. T., Xu, S., Mercurio, F., Barbosa, M., Cobb, M. H. and Gaynor, R. B. (1998) HTLV-I Tax protein binds to MEKK1 to stimulate IkappaB kinase activity and NF-kappaB activation. Cell 93, 875-884.

Yoshida, H., Kato, N., Shiratori, Y., Otsuka, M., Maeda, S., Kato, J. and Omata, M. (2001) Hepatitis C virus core protein activates nuclear factor kappa B-dependent signaling through tumor necrosis factor receptor-associated factor. J. Biol. Chem. 276, 16399-16405. 